samtaktivierung pro Flächeneinheit

$C_{0}(\delta)=\int_{0}^{\delta} c_{0}(x, \delta) \mathrm{d} x=v n \mu_{\mathrm{act}} \delta\left\{\frac{\Phi_{0}(\mu \delta)}{2 \mu \delta}\right.$ $\left.+\mu_{\mathrm{s}} \delta \frac{\chi(\mu \delta)}{2 \mu \delta}\right\}$

$\left(\mu=\mu_{\mathrm{a}}+\mu_{\mathrm{B}}\right)$, wobei die von Botнe ${ }^{7}$ angegebene Funktion

$\Phi_{0}(t)=1-2 E_{3}(t)=1-(1-t) e^{-t}+t^{2} \quad \operatorname{Ei}(-t)$

ist und das Integral

$\chi(t)=\frac{1}{2 t} \int_{0}^{t}\left\{E_{2}(y)+E_{2}(t-y)\right\}$

$$
\cdot\left\{2-E_{2}(y)-E_{2}(t-y)\right\} \mathrm{d} y
$$

numerisch bestimmt werden mußte (s. Abb. 21).

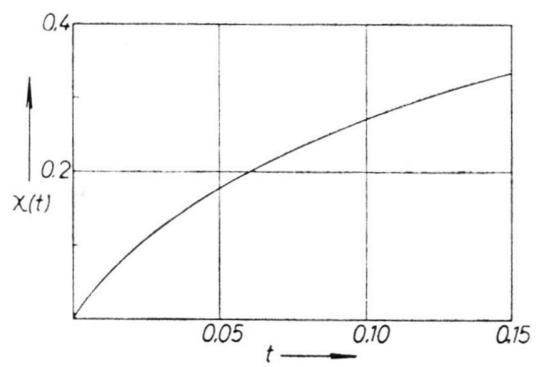

Abb. 21. Die Funktion $\chi(t)$.
Die für monoenergetische Neutronen abgeleitete Gl. (35) ist bei Anwendung auf ein thermisches Neutronenfeld über die Maxwell-Verteilung zu mitteln. Für eine $1 / v$-Abhängigkeit von $\sigma_{\mathrm{a}}$ wurde diese Mittelung in einer früheren Arbeit durchgeführt ${ }^{10}$. Danach gilt Gl. (35) in sehr guter Näherung (auf $0,3 \%$ bei $\mu_{\mathrm{a}} \delta<0,1$ ), wenn für $v$ und $\mu_{\mathrm{a}}$ die Werte bei der wahrscheinlichsten Geschwindigkeit $v_{0}$ der MAxwelL-Verteilung eingesetzt werden. Für die mittlere spezifische Aktivierung $\bar{c}(\delta)=C(\delta) / \delta$ ergibt sich somit unter Berücksichtigung der Aktivierungsstörung $\varkappa_{\mathrm{C}}=\left(C_{0}-C\right) / C$ aus Gl. (35)

$\bar{c}(\delta)=v_{0} n \mu_{\mathrm{act}} \frac{1}{1+\varkappa_{\mathrm{C}}}\left\{\frac{\Phi_{0}(\mu \delta)}{2 \mu \delta}+\mu_{\mathrm{s}} \delta \frac{\chi(\mu \delta)}{2 \mu \delta}\right\}$

Damit ist Gl. (7) aus Abschnitt 2 hergeleitet.

Für $\mu_{\mathrm{s}}=0$ geht unser Ergebnis Gl. (35) in die von Bотне $^{7}$ angegebene Gl. (6) über. Die Berücksichtigung der Streuung in Gl. (35) bringt gegenüber der Gl. (6) von Bотне nur eine geringfügige Erhöhung der Aktivierung mit sich, die für unsere Folien $1 \%$ nicht übersteigt. Dies läßt sich leicht plausibel machen: die mittlere Weglänge in der Sondensubstanz wird für senkrecht auftreffende Neutronen durch die Streuung vergrößert, für die schräg einfallenden dagegen verkleinert, wobei sich beide Effekte weitgehend kompensieren.

Herrn Prof. WIRTz danke ich für die Anregung zu dieser Arbeit und sein stets förderndes Interesse.

Für die $\beta$ - $\gamma$-Koinzidenzmessung stellte mir freundlicherweise Herr H. Houtermans vom Isotopenlaboratorium Göttingen seine Koinzidenzapparatur zur Verfügung. Ihm und Herrn D. H. Vincent habe ich außerdem für wertvolle Hinweise zu danken.

\title{
Über die Einfänge negativer K-Mesonen durch zusammengesetzte Kerne
}

\author{
Von W. Alles
}

Aus dem Max-Planck-Institut für Physik, Göttingen *

(Z. Naturforschg. 13 a, 740-745 [1958]; eingegangen am 20. Mai 1958)

\begin{abstract}
Es werden die Wechselwirkungen der negativen K-Mesonen mit den zusammengesetzten Kernen der photographischen Emulsion analysiert. Die $\Sigma^{-}$-Einfangsterme werden studiert; $75 \%$ hiervon weisen keine geladenen Sekundärspuren auf. Das mittlere Covroms-Potential der die $\mathrm{K}^{-}$-Mesonen einfangenden Kerne beträgt $(8,4 \pm 2) \mathrm{MeV}$. - Aus dem Energiespektrum der $\Sigma$-Hyperonen wird ein anziehendes $\Sigma$-Kernpotential $V_{\Sigma}=-(15 \pm 10) \mathrm{MeV}$ gefolgert. Die Emissionshäufigkeit der $\Sigma$-Hyperonen liefert Aussagen über die $\Sigma$-Nukleon-Wirkungsquerschnitte.
\end{abstract}

In dieser Arbeit werden einige Eigenarten des Einfangs negativer K-Mesonen in photographischer Kernemulsion untersucht. Wir befassen uns hauptsächlich mit solchen Ereignissen, bei denen ein geladenes Hyperon und ein entgegengesetzt geladenes $\pi$-Meson ohne Emission weiterer geladener Teilchen erzeugt werden (,$\Sigma \pi^{\prime}$-Ereignisse). Diese Ereignisse sind vermutlich $\mathrm{K}^{-}$-Einfänge durch gebundene Protonen nahe der Oberfläche der Kerne mit keinen

* Jetzt am Istituto di Fisica dell'Università, Bologna. oder nur geringfügigen sekundären Wechselwirkungen:

$$
\mathrm{K}^{-}+\mathrm{p} \rightarrow \Sigma^{ \pm}+\pi^{\mp} .
$$

Hieraus werden Aussagen über das Kernpotential des $\Sigma$-Hyperons, die mittlere freie Weglänge der Hyperonen in Kernmaterie, die Einfangshäufigkeiten in den schweren und leichten Emulsionskernen, sowie über die Häufigkeit der $\Sigma^{-}$-Hyperonen, die ohne Emission geladener Teilchen eingefangen werden $\left(\Sigma_{2}^{-}\right)$, gewonnen. Zu diesem Zweck benutzen 


\begin{tabular}{|c|c|c|c|c|c|c|c|c|}
\hline & $0-5$ & $5-10$ & $10-20$ & $20-30$ & $30-40$ & $40-50$ & $50-60$ & zusam. \\
\hline$\Sigma^{+} \rightarrow \mathrm{p}+\pi^{0} \quad$ in Ruhe & 1,5 & 7 & 9 & 8 & 3,5 & 0 & 0 & 29 \\
\hline$\Sigma^{+} \rightarrow \mathrm{n}+\pi^{+} \quad$ in Ruhe & 0 & 2 & 8,5 & 7,5 & 5 & 1 & 1 & 25 \\
\hline$\Sigma^{+} \rightarrow \mathrm{p}+\pi^{0} \quad$ im Fluge & 0 & 2 & 3 & 4 & 0 & 1 & 1 & 11 \\
\hline 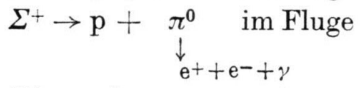 & 0 & 0 & 0 & 0 & 1 & 0 & 0 & 1 \\
\hline$\Sigma^{ \pm} \rightarrow \pi^{ \pm}+\mathrm{n} \quad$ im Fluge & 0,5 & 2,5 & 8 & 3 & 7 & 2 & 1 & 24 \\
\hline Stern im Fluge & 0 & 0 & 0 & 1 & 0 & 0 & 0 & 1 \\
\hline $\begin{array}{l}\text { im Fluge verschwindende } \\
\text { Teilchen }\end{array}$ & 0 & 0 & 2 & 1 & 0 & 1 & 0 & 4 \\
\hline$\Sigma$--Sterne in Ruhe & 2 & 3 & 6 & 6 & 1 & 1 & 0 & 19 \\
\hline $\begin{array}{l}\Sigma-\text { mit AUGER-Elektron in } \\
\text { Ruhe }\end{array}$ & 3,5 & 3,5 & 5 & 0 & 1 & 0 & 0 & 13 \\
\hline „,Protonen“ mit blob & 0 & 2 & 7 & 2,5 & 1,5 & 0 & 0 & 13 \\
\hline ,Protonen“" & 39 & 29 & 21,5 & 15,5 & 23 & 15,5 & 4,5 & 148 \\
\hline
\end{tabular}

Tab. 1. Energieintervalle in MeV.

wir für die Wechselwirkungen von $\mathrm{K}^{-}$-Mesonen in den zusammengesetzten Kernen der photographischen Emulsion ein Modell, das dem von Gilbert u. a. ${ }^{1}$ aufgestellten analog ist.

In diesem Experiment wurde ein Block von 150 je $600 \mu$ dicken Schichten einer photographischen Kernemulsion (Ilford G-5) mit den Gesamtabmessungen $9 \mathrm{~cm} \times 17,5 \mathrm{~cm} \times 15 \mathrm{~cm}$ durchmustert, der dem doppelt fokussierten $300 \mathrm{MeV} / \mathrm{c}-\mathrm{K}^{-}$-Strahl des Bevatrons in $\mathrm{Berkeley}{ }^{2}$ ausgesetzt worden war. Nach der Methode der „Durchmusterung längs der Spuren" wurden 3300 Spuren verfolgt, deren Flugbahnen um weniger als $5^{\circ}$ von der mittleren Richtung der Bahnen der $\mathrm{K}^{-}$-Mesonen abwichen und deren Ionisation ungefähr der 2,5-fachen Minimumionisation entsprach. $2622 \mathrm{~K}^{-}$-Mesonen kamen zur Ruhe. Der Protonenuntergrund war sehr klein (ca. $2 \%$ ).

In einer früheren Veröffentlichung ${ }^{3}$ wurden die Flugwechselwirkungen dieser $\mathrm{K}^{-}$-Mesonen untersucht.

\section{Experimentelle Ergebnisse}

Aus den 2622 Ruheeinfängen von $\mathrm{K}^{-}$-Mesonen durch zusammengesetzte Kerne wählten wir diejenigen 275 Prozesse aus, in denen ein geladenes $\pi$ Meson und ein geladenes Baryon emittiert wurden. Da 10\% der Platten unseres Blocks nicht sehr durchsichtig waren, konnten im allgemeinen weder die Spuren der $\pi$-Mesonen verfolgt werden, noch konn-

\footnotetext{
1 F. C. Gilbert, Ch. E. Violet u. R. S. White, UCRL-4814 und Phys. Rev. 107, 228 [1957].

2 W. H. Barkas, Rochester Konferenz 1957.
}

ten an ihnen zuverlässige Energiemessungen und Bestimmungen des Ladungsvorzeichens durchgeführt werden. Die Spuren der Teilchen mit baryonischer Masse wurden bis zu ihrem Ende verfolgt; die Ergebnisse zeigt Tab. 1.

Die beobachtete $\Sigma$-Spurlänge von nur $12,9 \mathrm{~cm}$ (ungefähr die halbe geometrische freie Weglänge) läßt vermuten, daß es sich bei den 4 im Fluge verschwindenden Teilchen um Zerfälle $\Sigma^{ \pm} \rightarrow \mathrm{n}+\pi^{ \pm}$ handelt, bei denen die Zerfalls- $\pi$-Mesonen nicht beobachtet wurden. Aus dem wohlbekannten Verzweigungsverhältnis der $\Sigma^{+}$-Hyperonen folgt, daß die so auf 28 erhöhte Anzahl von $\Sigma^{ \pm} \rightarrow \mathrm{n}+\pi^{ \pm}$sich aus ungefähr 12 Zerfällen der Art $\Sigma^{+} \rightarrow \mathrm{n}+\pi^{+}$und 16 der Art $\Sigma^{-} \rightarrow \mathrm{n}+\pi^{-}$zusammensetzt. Der zweistrahlige Stern hat eine Primärenergie von $23 \mathrm{MeV}$ und eine sichtbare Sekundärenergie von $63 \mathrm{MeV}$. Es handelt sich also hier nicht um einen Protonenstern, sondern um einen Stern, der von einem $\Sigma^{ \pm}$-Hyperon erzeugt wird, dessen Ladungsvorzeichen aber unbestimmt bleibt.

Die Gesamtzahl der emittierten $\Sigma^{+}$-Hyperonen ist also 81. Hier haben wir für einen 10-proz. Verlust von Ruhezerfällen $\Sigma^{+} \rightarrow \mathrm{n}+\pi^{+}$korrigiert. Die Gesamtzahl der emittierten $\Sigma^{-}$-Hyperonen werden wir im folgenden Abschnitt zu 92,5 abschätzen. Also ist das Verhältnis der negativen zu den positiven, den Kern verlassenden Hyperonen, die an gebundenen Protonen erzeugt wurden, $\Sigma^{-} / \Sigma^{+}=92,5 / 81=(1,14$ $\pm 0,19)$.

3 W. Alles, N. N. Biswas, M. Ceccharelli u. J. Crussard, Nuovo Cim. 6, 571 [1957]. 
In Abb. 1 ist das Energiespektrum von 66,5 $\Sigma^{+}$. Hyperonen aufgetragen. Um die Flugzerfälle der Art $\Sigma^{+} \rightarrow \pi^{+}+\mathrm{n}$ zu berücksichtigen, hat man hierfür unter der Annahme $\tau_{\Sigma+}=1,13 \cdot 10^{-10} \mathrm{sec}$ korrigiert. In Abb. 2 ist das Energiespektrum von 32,5 in die-

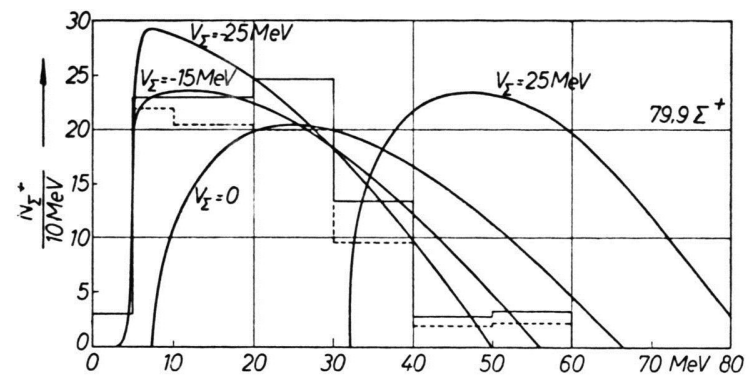

Abb. 1. $N_{\Sigma+} / 10 \mathrm{MeV}$ für verschiedene Kernpotentiale $V_{\Sigma}$. mit Korrektur; - - ohne Korrektur.

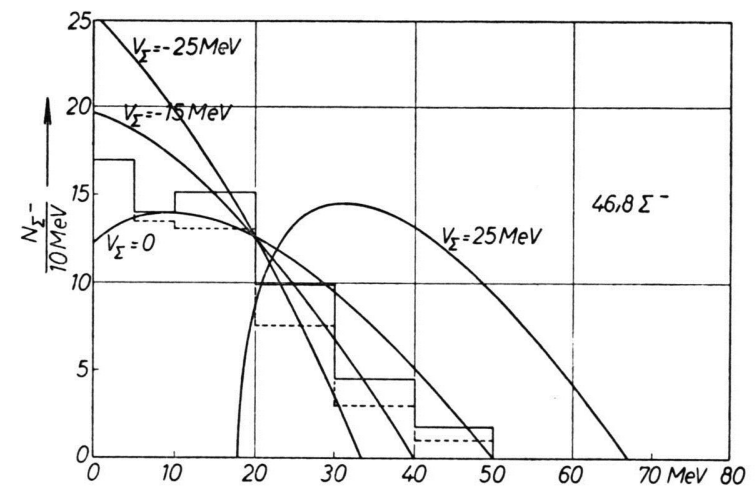

Abb. 2. $N_{\Sigma-} / 10 \mathrm{MeV}$ für verschiedene Kernpotentiale $V_{\Sigma}$. mit Korrektur; - - o ohne Korrektur.

ser Arbeit identifizierten $\Sigma^{-}$-Hyperonen zusammen mit 7 von Gilbert u. a. ${ }^{1}$ gefundenen aufgetragen. Hier wurde für die Zerfälle $\Sigma^{-} \rightarrow \pi^{-}+$n korrigiert unter der Annahme von $\tau_{\Sigma^{-}}=1,90 \cdot 10^{-10} \mathrm{sec}$.

Bei den in Tab. 1 mit "Protonen“ bezeichneten Teilchen handelt es sich um eine Mischung von Protonen und $\Sigma^{-}$-Hyperonen, die ohne Emission geladener Sekundärspuren enden. Die Identifikation der „Protonen“ mit blob als $\Sigma_{o}^{-}$'s, die mit einem kleinen Rückstoß enden, ist sehr zweifelhaft. Eine Durchmusterung von 300 Protonenspuren, die in einem mit $\mathrm{K}^{+}$-Mesonen und Protonen beschossenen Block durchgeführt wurde, dessen Elektronen-Untergrund mit dem unseres Blockes vergleichbar ist, ergab, daß 13\% der Enden von Protonenspuren diese Art von „irregulärem“ blob aufweisen. Das stimmt sehr gut mit der gegenwärtigen Anzahl von $\Sigma_{0}{ }^{-}$'s und Protonen überein. Daher werden diese im folgenden zusammen mit den „Protonen“ behandelt.

\section{Abschätzung der Anzahl der $\Sigma_{Q}^{-}$-Einfänge in Ruhe}

Wir wollen die Anzahl der $\Sigma_{Q}^{-}$s $\mathrm{s}$ mit Hilfe der Winkelverteilung der $\Sigma \pi$ - und der "Proton“ $\pi$-Ereignisse abschätzen. In Abb. 3 sind beide aufgeführt. $\Theta$ ist der Raumwinkel zwischen dem $\Sigma$-Hyperon bzw. dem Proton und dem $\pi$-Meson. Die 120 hier

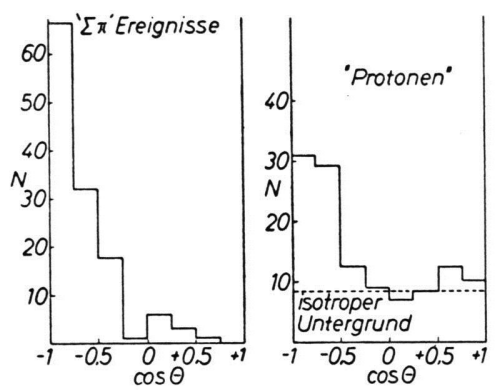

Abb. 3. Verteilung für den Winkel $\Theta$ zwischen zwei Spuren.

aufgetragenen „Protonen“ erfüllen $100 \mu \leqq R(p)$ $\leqq 8,500 \mu$. Man fand keine Hyperonen mit Reichweiten größer als $8,500 \mu$, und mit dem hochenergetischen Abschneiden beseitigte man eine zusätzliche Kontamination durch Protonen.

In Abb. 3 ist deutlich zu erkennen, daß die $\Sigma \pi$ Ereignisse erwartungsgemäß bei $\Theta=180^{\circ}$ ein scharfes Maximum haben. Auf der anderen Seite würde man für die Verdampfungsprotonen eine annähernd isotrope Verteilung erwarten, falls der Anregungsprozeß nicht sehr einseitig an der Oberfläche konzentriert ist. Wenn wir diese Isotropie voraussetzen, kann man leicht ableiten, daß die 120 ausgewählten „Protonen“ sich aus 68 isotrop verteilten Verdampfungsprotonen und 52 um $\Theta=180^{\circ}$ konzentrierten Ereignissen zusammensetzen. Der Vergleich der Winkelverteilung der $\Sigma \pi$-Ereignisse und der breiteren Verteilung dieser 52 Ereignisse zeigt, daß diese sich aus ungefähr 8,5 Protonen, die z. B. durch die Prozesse $\quad \Sigma^{+}+\mathrm{n} \rightarrow \Sigma^{0}+\mathrm{p} \quad$ oder $\quad \Lambda^{0}+\mathrm{p} \rightarrow \Lambda^{0}+\mathrm{p}$ aus dem Kern herausgestoßen worden sind, und 43,5 $\Sigma$-Hyperonen zusammensetzen. Nach Korrektion für die niedrigenergetischen $\Sigma^{-}$-Hyperonen und die Ruhezerfälle $\Sigma^{+} \rightarrow \pi^{+}+\mathrm{n}$, bei denen die $\pi^{+}$. Mesonen nicht beobachtet wurden, erhält man schließlich für die Anzahl der negativen Hyperonen, die weder mit einem Stern noch mit einem AugerElektron enden, $\Sigma_{\varrho}^{-}=(46 \pm 14,5)$. Der hier angegebene Fehler hat seine Ursache nur in den statistischen Unsicherheiten der Winkelverteilungen der 
$\Sigma \pi$ - und der "Proton" $\pi$-Ereignisse, sowie in den zwei Korrektionstermen.

Eine unabhängige Methode geht von der Lebensdauer der $\Sigma^{-}$-Hyperonen aus, wie sie in der Blasenkammer von Alvarez u. a. ${ }^{4}$ gemessen wurde:

$$
\tau_{\Sigma^{-}}=(1,6 \pm 0,2) 10^{-10} \mathrm{sec} .
$$

Bei dieser Methode wird unter der Annahme, daß die $\Sigma_{Q}^{-}$dieselbe Energieverteilung aufweisen wie die identifizierten $\Sigma^{-}$-Hyperonen, die gesamte Flugdauer der negativen Hyperonen so eingerichtet, daß sie die richtige Lebensdauer der $\Sigma^{-}$ergibt. Es folgt $\Sigma_{0}{ }^{-}=(38 \pm 24)$. Ein gewichtetes Mittel beider Ergebnisse führt zu $\Sigma_{\ell}^{-}=(44 \pm 12)$.

Fassen wir zusammen: 32,5 identifizierte $\Sigma^{-}$, 16 abgeschätzte Zerfälle $\Sigma^{-} \rightarrow \pi^{-}+\mathrm{n}, 44$ abgeschätzte $\Sigma_{Q}^{-}$; also ist die Gesamtzahl der emittierten $\Sigma^{-}$-Hyperonen 92,5 .

\section{Der $\Sigma^{-}$-Einfangprozeß}

In Abb. 4 ist die Häufigkeitsverteilung der Spurenzahl der $76 \Sigma^{-}$-Hyperon-Einfangsterne aufgetragen. In die Gruppe der 0-strahligen Sterne fallen 57, also

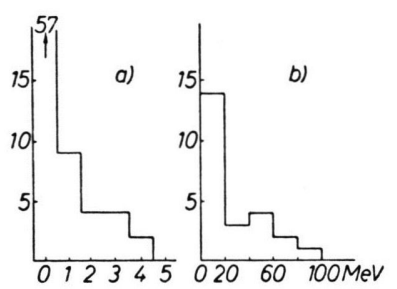

Abb. 4. $\Sigma^{-}$-Einfang-Sterne. a) Häufigkeitsverteilung der Spurenzahl. b) Verteilung der sichtbaren Energie.

75\%. Gilbert u. a. ${ }^{1}$ und Fry u. a. ${ }^{5}$ haben die Häufigkeit dieser 0-strahligen Sterne aus der Energieverteilung der „Proton“ $\pi$-Ereignisse bzw. der Häufigkeit der Auger-Elektronen zu 61\% bzw. 57\% abgeschätzt. Ein gewichtetes Mittel der drei Ergebnisse führt auf $\Sigma_{0}^{-} / \Sigma^{-}=(66 \pm 10) \%$. Der Fehler rührt hier von den systematischen Unsicherheiten der angewandten Methoden her.

In Abb. 4 ist auch die Verteilung der sichtbaren Energie von $24 \Sigma^{-}$-Einfängen aufgetragen. Nur ein Ereignis mit einer höheren Energie als $80 \mathrm{MeV}$ wurde beobachtet.

${ }^{4}$ L. W. Alvarez, H. Bradner, P. Falk-Vairant, J. D. Gow, A. H. Rosenfeld, F. T. Solmitz u. R. D. Tripp, UCRL-3583 und Nuovo Cim. 5, 1026 [1957] und UCRL-3775.
Der Einfangprozeß des negativen $\Sigma$-Hyperons führt primär auf ungeladene Produkte:

$$
\Sigma^{-}+\mathrm{p} \rightarrow \mathrm{n}+\begin{gathered}
\Lambda^{0}+81 \mathrm{MeV} \\
\Sigma^{0}+7 \mathrm{MeV}
\end{gathered} .
$$

Der zweite Prozeß wird aber durch das Pauli-Prinzip praktisch verboten. Falls das $\Lambda^{0}$-Hyperon im Kern eingefangen wird, wird es entweder den Prozeß $\Lambda^{0} \rightarrow \mathrm{N}+\pi$ oder den Proze $\beta \Lambda^{0}+\mathrm{N} \rightarrow \mathrm{n}+\mathrm{N}$ eingehen, die beide auf gut erkennbare Sterne führen. Die Energien der erzeugten $\Lambda^{0}$-Hyperonen erstrecken sich aber innerhalb des Kernes von $26 \mathrm{MeV}$ bis $94 \mathrm{MeV}$, während das $\Lambda^{0}$-Kernpotential ungefähr $20 \mathrm{MeV}$ beträgt. Daher erwartet man wenige $\Lambda^{0}$. Einfänge, da diese nur durch die geringfügigen sekundären $A^{0}$-Nukleon-Streuungen verursacht werden könnten.

\section{Wechselwirkungsmodell}

In den hier studierten Ereignissen werden ein geladenes Hyperon und ein geladenes $\pi$-Meson beobachtet. Daher handelt es sich um die Reaktionen ${ }^{5}$

$$
\mathrm{K}^{-}+\mathrm{p} \rightarrow \Sigma^{+}+\pi^{-}+103 \mathrm{MeV}
$$

und

$$
\mathrm{K}^{-}+\mathrm{p} \rightarrow \Sigma^{-}+\pi^{+}+96 \mathrm{MeV}
$$

falls kein wichtiger Beitrag von Ladungsaustauschungen vorhanden ist. Wir nehmen an, daß die Reaktion in einem Potentialtopf der Tiefe $\left(V_{\Sigma}+V_{\mathrm{c}}\right)$ stattfindet. $V_{\Sigma}$ ist das $\Sigma$-Kernpotential und $V_{\mathrm{c}}$ das Coulomb-Potential. Das Energiespektrum der Hyperonen ist durch die obigen Reaktionen (1) und (2) unter Berücksichtigung der Anregungsenergie $E_{\mathrm{A}}$ des Kernes und der Bindungsenergie $E_{\mathrm{B}}$ des Protons, sowie der Impulsverteilung der Protonen bestimmt. Gilbert u. a. ${ }^{1}$ haben für $E_{\mathrm{A}}+E_{\mathrm{B}} \sim 20 \mathrm{MeV}$ erhalten. Für die Impulsverteilung der Protonen haben wir ein völlig entartetes F ERMI-Gas mit einem mittleren Impuls von $160 \mathrm{MeV} / \mathrm{c}$ angenommen. Für das $\pi$-Mesonen-Kernpotential haben wir $V_{\pi}=-40 \mathrm{MeV}$ gesetzt $^{6}$. Für die 4 Werte $V_{\Sigma}=25,0,-15$ und $-25 \mathrm{MeV}$ wurden die $\Sigma$-Energiespektren berechnet. Während diese von $V_{\pi}$ nicht stark abhängen (eine Änderung in $V_{\pi}$ von $5 \mathrm{MeV}$ verursacht eine Verschiebung in den Spektren der Pionen und Hyperonen von nur $0,5 \mathrm{MeV}$ ), sind sie von $V_{\Sigma}$ voll abhän-

5 W. F. Fry, J. Schneps, G. A. Snow, M. S. Swami u. D. C. Wold, Phys. Rev. 107, 257 [1957].

${ }^{6}$ R. M. Frank, J. L. Gammel u. K. M. Watson, Phys. Rev. 101, 891 [1956]. 
gig. Für das Coulomb-Potential haben wir das im nächsten Abschnitt bestimmte $V_{\mathrm{c}}=8 \mathrm{MeV}$ angenommen. Ferner nehmen wir an, daß: 1. die Winkelverteilung der Reaktionen (1) und (2) im Schwerpunktsystem isotrop ist, 2. die negativen und positiven Hyperonen, deren Energie innerhalb des Kernes gröBer als $-\left(V_{\Sigma}+V_{\mathrm{c}}\right)$ bzw. $-V_{\Sigma}$ ist, den Kern verlassen, 3. der Impuls des Protons ganz auf die beiden Reaktionsprodukte übertragen wird, 4. der Impuls des $\mathrm{K}^{-}$-Mesons gleich Null ist, 5. die erzeugten Hyperonen keine Streuungen erleiden, bevor sie aus dem Kern herauskommen. Die Gültigkeit der Annahmen 4. und 5. ist zweifelhaft. Capps ${ }^{7}$ hat den Effekt der Reflektion an der Oberfläche des Potentialtopfes berechnet. Es ergibt sich, daß Annahme 2. für $V_{\Sigma} \geqq-15 \mathrm{MeV}$ vernünftig ist. Die so berechneten Hyperonenspektren sind in Abb. 1 und 2 zusammen mit den experimentellen Spektren wiedergegeben. Der Vergleich beider Spektren zeigt, daß das $\Sigma^{+}$. sowie das $\Sigma^{-}$-Spektrum mit einem abstoßenden $\Sigma$. Kernpotential nicht verträglich sind. Eine gute Übereinstimmung erhält man mit $V_{\Sigma}=-(15 \pm 10) \mathrm{MeV}$

\section{Das mittlere Coulomb-Potential}

Aus Abschnitt 2 folgt, daß das Verhältnis der negativen zu den positiven den Kern verlassenden Hyperonen, die durch $\mathrm{K}^{-}$-Mesonen an gebundenen Protonen erzeugt wurden, $\Sigma^{-} / \Sigma^{+}=(1,14 \pm 0,17)$ ist, gegenüber einem Verhältnis $\Sigma^{-} / \Sigma^{+}=(1,91 \pm 0,31)$, das von Alvarez u. a. ${ }^{4}$ an freien Protonen gemessen wurde. Unter Voraussetzung der Ladungsunabhängigkeit für das $\Sigma$-Kernpotential, d.h. $V_{\Sigma+}=$ $V_{\Sigma_{-}}$, ist dieser Unterschied mit den Annahmen des vorigen Abschnittes leicht zu erklären. Während die negativen Hyperonen durch das Coulomb-Potential $V_{c}$ in den Bereich niedrigerer Energien verschoben werden, werden die positiven Hyperonen durch dasselbe Potential auf höhere Energien gebracht. Ferner ist infolge der Massendifferenz beider Hyperonen die kinetische Energie des positiven Hyperons um 1,4 $\mathrm{MeV}$ höher als die des negativen. Also entsprechen die $\Sigma^{-}$-Hyperonen, deren Energie größer als 0 ist (in bezug auf das Energiespektrum bei der Erzeugung im Kern), solchen $\Sigma^{+}$-Hyperonen, deren Energie größer als $\left(2 V_{\mathrm{c}}+1,4\right)$ ist. Sodann erhalten

7 R. H. CAPps, Phys. Rev. 107, 239 [1957].

8 R. E. Marshak, Meson Physics, S. 170-171 [1952].

9 S. Gasionowicz, UCRL-3074.

10 B. Hahn, D. G. Ravenhall u. R. Hofstadter, Phys. Rev. 101, 1131 [1956]. wir $V_{\mathrm{c}}=(8,4 \pm 2) \mathrm{MeV}$. Dies führt zu Einfangshäufigkeiten in den schweren $(\mathrm{Ag}, \mathrm{Br})$ und leichten (C, N, O) Emulsionskernen von $(73 \pm 29) \%$ bzw. $(27 \pm 29) \%$, die gut mit den bekannten Häufigkeiten für $\pi^{-}$-Mesonen $(60-70 \%$ bzw. $40-30 \%)$ übereinstimmen ${ }^{8}$.

\section{Die Absorption geladener Hyperonen}

Um Hinweise hierüber zu bekommen, muß man selbstverständlich alle $\mathrm{K}^{-}$-Einfänge berücksichtigen und nicht nur die hier analysierten,$\Sigma \pi$ "-Ereignisse. Aus den Ergebnissen von $F_{R Y}$ u. a. ${ }^{5}$ folgt, nach Korrektion für $\mathrm{K}_{2}^{-}$und für solche Einfänge, bei denen nur ein $\pi$-Meson emittiert wird ${ }^{3}$, daß in $5,9 \%$ bzw. 9,9\% aller $\mathrm{K}^{-}$-Einfänge ein $\Sigma^{+}$- bzw. ein $\Sigma^{-}$-Hyperon emittiert wird. Unter Annahme der Ladungsunabhängigkeit kann man aus den Blasenkammerergebnissen leicht die Erzeugungshäufigkeiten der $\Sigma^{+}$- und $\Sigma^{-}$-Hyperonen berechnen ${ }^{9}$. Nach Korrektion für den Neutronenüberschuß der schweren Kerne folgt, daß in $14,6 \%$ bzw. $43,1 \%$ aller $\mathrm{K}^{-}$-Einfänge ein $\Sigma^{+}$bzw. ein $\Sigma^{-}$erzeugt wird. Also werden $39,5 \%$ bzw. $23 \%$ der erzeugten positiven bzw. negativen Hyperonen aus dem Kern emittiert.

Diese Emissionswahrscheinlichkeit ist das Produkt aus der Wahrscheinlichkeit $p$, dem Einfang durch den Potentialtopf zu entgehen, und der Wahrscheinlichkeit $\gamma$, ohne Wechselwirkung den Kernrand zu erreichen. Wir werden im folgenden $V_{\Sigma}=-15 \mathrm{MeV}$ annehmen. Dann folgt aus dem in Abschnitt 4 beschriebenen Modell, daß $p_{\Sigma+}=81 \%$ und $p_{\Sigma-}=57 \%$ ist. Also ist $\gamma \Sigma^{+}=0,49$ und $\gamma \Sigma_{-}=0,40$.

Diese Wahrscheinlichkeit $\gamma$ ist eine Funktion der mittleren freien Weglänge $\lambda$ des Hyperons in Kernmaterie und des Ortes des einfangenden Nukleons im Kern. Für den letzteren nehmen wir eine gleichmäßige Verteilung im Kern an. Dann ist

$$
\begin{aligned}
\gamma=\frac{1}{4 \pi V} \int_{\dot{\Omega}, V} e^{-d / \lambda} \mathrm{d} \Omega \mathrm{d} V \\
\quad=\frac{3 \hat{\lambda}^{3}}{8 R^{3}}\left\{\frac{2 R^{2}}{\lambda^{2}}-1+e^{-2 R / \lambda}\left(\frac{2 R}{\lambda}+1\right)\right\},
\end{aligned}
$$

mit $R=1,2 \cdot 10^{-13} \cdot \mathrm{A}^{1 / 3} \mathrm{~cm}^{10}$. Aus den obigen $\gamma$ folgt dann

$\lambda_{\Sigma^{+}}=4,15 \cdot 10^{-13} \mathrm{~cm}$ und $\lambda_{\Sigma-}=3,4 \cdot 10^{-13} \mathrm{~cm}$.

Zur Absorption der geladenen Hyperonen tragen die Prozesse

$$
\Sigma \pm+{ }_{\mathrm{p}}^{\mathrm{n}} \rightarrow A^{0}+{ }_{\mathrm{n}}^{\mathrm{p}} \text { und } \Sigma \pm+{ }_{\mathrm{p}}^{\mathrm{n}} \rightleftarrows \Sigma^{0}+{ }_{\mathrm{n}}^{\mathrm{p}}
$$

(Wirkungsquerschnitte $\sigma_{.1}$ bzw. $\sigma_{\mathrm{LA}}$ ) 
und diejenigen Streuungen

$$
\Sigma^{ \pm}+{ }_{\mathrm{p}}^{\mathrm{n}} \rightarrow \Sigma^{ \pm}+{ }_{\mathrm{p}}^{\mathrm{n}} \quad \text { und } \quad \Sigma^{ \pm}+{ }_{\mathrm{n}}^{\mathrm{p}} \rightarrow \Sigma^{ \pm}+{ }_{\mathrm{n}}^{\mathrm{p}}
$$

(Wirkungsquerschnitte $\sigma_{\text {str }}^{1}$ bzw. $\sigma_{\text {str }}^{2}$ )

bei, die die Energie des Hyperons so stark vermindern, daß es den Kern nicht mehr verlassen kann; der Anteil dieser Streuungen sei $f$. Aus der Ladungsunabhängigkeit dieser Reaktionen folgt unmittelbar die Gleichheit der mit einem Symbol gekennzeichneten Wirkungsquerschnitte und das Verhältnis

$$
\frac{\sigma_{\mathrm{str}}}{\sigma_{\mathrm{LA}}}>0,5 \quad \text { mit } \quad \sigma_{\mathrm{str}}=\frac{\sigma_{\mathrm{str}}^{1}+\sigma_{\mathrm{str}}^{2}}{2} .
$$

Nach Berücksichtigung des PAuli-Prinzips ${ }^{11}$ und Abschätzung von $f$ aus den Montecarlo-Rechnungen

11 R. Sternheimer, Phys. Rev. 106, 1027 [1957].

12 N. N. Biswas u. M. Ceccarelli, private Mitteilung. von Biswas und Ceccarelli ${ }^{12}$ folgt

$$
\sigma_{.1}+0,53 \sigma_{\mathrm{LA}}+1,06 \sigma_{\mathrm{str}}=39 \mathrm{mb} .
$$

Nach den Blasenkammer-Ergebnissen über $\Sigma^{-}$-Einfänge in Ruhe ist $\sigma_{\mathrm{LA}} \sim \frac{3}{4} \sigma_{\Lambda}$ eine vernünftige Annahme. Es folgt dann: $\sigma_{\Lambda}<22 \mathrm{mb}, \sigma_{\mathrm{LA}}<16,5 \mathrm{mb}$ und $8,5 \mathrm{mb}<\sigma_{\text {str }}<37 \mathrm{mb}$ für eine mittlere $\Sigma$. Hyperon-Energie außerhalb des Kernes von $20 \mathrm{MeV}$.

Herrn Prof. W. Heisenberg und Herrn Prof. K. Wirtz bin ich für die freundliche Aufnahme im Max-PlanckInstitut für Physik zu Dank verpflichtet. Den Herren N. N. Biswas, J. Crussard, K. Gottstein und besonders M. Ceccarelli danke ich für zahlreiche fördernde Diskussionen. Herrn J. TIETGE für die Durchsicht dieser Arbeit. Dem Deutschen Akademischen Austauschdienst (Bonn) und der Facultad de Ingeniería (Montevideo) möchte ich für zwei Stipendien danken.

\title{
Zur Massenspektrometrie an Glimmentladungen
}

\author{
Von M. Pahl und U. Weimer \\ Aus der Forschungsstelle für Spektroskopie in der Max-Planck-Gesellschaft, Hechingen \\ (Z. Naturforschg. 13 a, 745-753 [1958] ; eingegangen am 13. Mai 1958)
}

\begin{abstract}
Der Anschluß eines richtungsfokussierenden $60^{\circ}$-Massenspektrometers an die stationäre positive Niederdruck-Säule zum Zwecke quantitativer Messungen von Ionenstromraten wird beschrieben. Die Ionengewinnung aus dem Plasma erfolgt durch ambipolare Effusion. Ionen, die stark verschiedene Beweglichkeiten besitzen, effundieren nicht mit gleicher Energie. Mittels einer Gegenfeldmethode werden solche Energiedifferenzen festgestellt und gemessen zwischen $\mathrm{Ne}^{+}-\mathrm{Ne}_{2}^{+}, \mathrm{Ne}^{+}-\mathrm{NeH}^{+}$, $\mathrm{He}^{+}-\mathrm{He}_{2}{ }^{+}$. Die Isotope des Neons haben innerhalb der erreichten Meßgenauigkeit gleiche Energie. Eine quantitative Abschätzung zeigt, daß Volumen-Rekombinationsprozesse unter den gewählten Bedingungen in He und Ne noch nicht merkbar werden.
\end{abstract}

Die massenspektroskopische Methode hat speziell bei Untersuchungen von Gasentladungen bisher nur geringe Anwendung gefunden ${ }^{1}$, obwohl sie mit am besten geeignet ist für die - zumindest qualitative Analyse der Ionen im Plasma. Abgesehen vom experimentellen Aufwand haben hauptsächlich wohl die folgenden Gründe methodischer Art einen vielfältigeren Einsatz der Massenspektroskopie in der Plasmaphysik verhindert:

1. Ein Massenanalysator herkömmlicher Art kann nicht im Innern eines ,ungestörten“ Plasmas arbei-

1 Abgesehen von Beobachtungen an Gasentladungen bei Experimenten mit vorwiegend massenspektroskopischer Zielsetzung sind uns die folgenden zitierten Arbeiten als solche bekannt, bei denen die Massenspektroskopie ausgesprochen zu Untersuchungen an Niederdruck-Plasmen eingesetzt worden ist: O. Luhr, Phys. Rev. 38, 1736 [1931]. - O. Luhr, Phys. Rev. 44, 459 [1933]. - J. L. Spencer-Smith, Phil. Mag. 19, 816, 1016 [1935]. - O. Luhr, J. Chem. Phys. 3, 146 [1935]. - O. Tüxen, Z. Phys. 103, 463 [1936]. J. W. Hiby, Ann. Phys., Lpz. (5) 34, 31 [1939]. - F. L. ten wie z. B. eine Langmuir-Sonde oder ein elektromagnetisckes Mikrowellenfeld. Die Ladungsträger müssen vielmehr irgendwie aus dem Plasma herausgeführt und können erst anschließend analysiert werden. Der Anschluß eines Massenspektrometers an ein Plasma muß für quantitative Mèssungen so beschaffen sein, daß zunächst das Plasma selbst durch die Entnahme relativ kleiner Ionenströme nicht gestört wird und daß zum anderen aus den im Massenspek. trometer gemessenen Strömen mit einiger Sicherheit auf die Vorgänge im Innern des Plasmas geschlossen

Arnot u. M. B. M'Ewen, Proc. Roy. Soc., Lond. A 171, 106 [1939]. - R. W. Lunt u. A. H. GREGG, Trans. Faraday Soc. 36, 1068 [1940]. - R. L. F. Boyd, Nature, Lond. 165, 142 [1950]. - R. L. F. Boyd, Proc. Phys. Soc., Lond. A 63, 543 [1950]. - J. A. Hornbeck u. J. P. Molnar, Phys. Rev. 84, 621 [1951]. - A. V. Phelps u. S. C. Brown, Phys. Rev. 86, 102 [1952]. - D. Morris, Proc. Phys. Soc., Lond. 68, 11 [1954]. - H. D. Beckey u. H. Dreeskamp, Z. Naturforschg. 9 a, 735 [1954]. - H. Dreeskamp, Z. Naturforschg. 12 a, 876 [1957]. 